\title{
Cobalt protoporphyrine IX-mediated heme oxygenase-I induction alters the inflammatory cytokine response, but not antigen presentation after experimental allogeneic bone marrow transplantation
}

\author{
PATRICIA EWING ${ }^{1 *}$, GERHARD C. HILDEBRANDT ${ }^{1 *}$, SIMONE PLANKE $^{1}$, \\ REINHARD ANDREESEN ${ }^{1}$, ERNST HOLLER ${ }^{1}$ and ARMIN GERBITZ ${ }^{2}$ \\ ${ }^{1}$ Department of Hematology and Oncology, University of Regensburg, Franz-Josef-Strauss-Allee 11, D-93053 Regensburg; \\ ${ }^{2}$ Department of Hematology and Oncology, University of Munich, Marcchioninistr.15, D-81377 Munich, Germany
}

Received March 19, 2007; Accepted May 19, 2007

\begin{abstract}
Acute graft-versus-host disease (aGvHD) remains the major cause of mortality after allogeneic stem cell transplantation. Acute GvHD can be partially prevented when heme oxygenase-1 (HO-1) is induced in the recipient prior to transplantation but the mechanisms are not fully understood. Using a murine haploidentical bone marrow transplantation (BMT) model $(\mathrm{C} 57 \mathrm{Bl} / 6 \rightarrow \mathrm{B} 6 \mathrm{D} 2 \mathrm{~F} 1)$ we tested whether HO-1 induction altered the alloreactive $\mathrm{T}$ cell response or rather modulated the inflammatory cytokine profile early after BMT. In vivo administration of cobalt protoporphyrine IX (CoPP) did not affect the expression of MHC class I and II or the costimulatory molecules CD80 and CD86 on murine peritoneal and on splenic dendritic cells (DCs). Allospecific $\mathrm{T}$ cell proliferation and interferon $\gamma$ secretion did not differ in mixed lymphocyte reactions using either CoPP-pretreated allogeneic recipients or control-treated DCs as stimulators. Furthermore, splenic DCs, isolated one to four days after BMT from CoPP-pretreated recipients did not show any differences in the expression of costimulatory molecules compared to untreated controls, and T cell expansion and the cytolytic capacity 14 days after BMT were equal in the control and CoPP-treated allogeneic groups. Serum tumor necrosis factor $\alpha$ levels were significantly reduced in CoPPtreated allogeneic recipients when compared to allogeneic
\end{abstract}

Correspondence to: Dr Patricia Ewing, Department of Hematology and Oncology, University of Regensburg, Medical School, FranzJosef-Strauss-Allee 11, D-93053 Regensburg, Germany

E-mail: patricia.ewing@klinik.uni-regensburg.de

${ }^{*}$ Contributed equally

Key words: allogeneic bone marrow transplantation, heme oxygenase-1, cobalt protoporphyrine, antigen presenting cells controls and did not differ from the syngeneic recipients. Our results indicate that the protective effects of CoPP-mediated HO-1 induction on survival and aGvHD after allogeneic BMT involve a reduction in the proinflammatory cytokine milieu rather than alteration in allospecific $\mathrm{T}$ cell stimulation.

\section{Introduction}

Allogeneic bone marrow transplantation is an important therapy for a number of malignant and nonmalignant hematopoietic disorders. Unfortunately its usage is limited by several complications including acute graft-versus-host disease (GvHD), which remains the major cause for transplantation-related mortality. Through previous research, it has become evident that host antigen-presenting cells (APCs) are critically involved by presenting alloantigens to donor $\mathrm{T}$ cells resulting in $\mathrm{T}$ cell activation (1). Activation of donor $\mathrm{T}$ cells results in proliferation and differentiation and leads to tissue damage in the aGvHD target organs liver, gut and skin $(2,3)$. In addition, cytokine secretion such as tumor necrosis factor $\alpha(\mathrm{TNF} \alpha)$ and interferon $\gamma(\mathrm{IFN} \gamma)$ by both host and donor immune cells contributes to this process $(4,5)$. Loss of gastrointestinal (GI) tract integrity and translocation of lipopolysaccharide (LPS) across the GI tract and into the circulation again promote further cytokine release and enhance the allostimulatory capacity of APCs (6).

The enzyme heme oxygenase (HO) catalyses the ratelimiting step in the degradation of heme into biliverdin, carbon monoxide $(\mathrm{CO})$ and free iron. Biliverdin is converted to bilirubin by biliverdin reductase, and free iron is directly sequestered by ferritin (7). To date, three isoforms of the enzyme are characterized. HO-1 is highly inducible in different cell types (e.g. endothelial cells, fibroblasts, smooth muscle cells and macrophages) by various cellular stress signals such as irradiation, endotoxins and nitric oxide $(7,8)$. $\mathrm{HO}-2$ is expressed constitutively and is present at high levels in the brain, testes and the vascular system (9), and HO-3 is also expressed in different organs such as the kidney, brain and heart. It has a lower affinity to heme than the other two and its function is unclear (10). Previous investigations 
suggest that the release of heme metabolites by HO-1 mediates anti-inflammatory and anti-apoptotic signals (11-13) and that HO- 1 induction results in reduced inflammation and apoptosis upon stress stimulation and prolonged graft survival in the case of solid organ transplantation (14-16). In addition, HO-1 seems cytoprotective against irradiation and against apoptosis-inducing cytokines $(17,18)$. Protoporphyrines are potent HO-1 inducers. Depending on the metal atom of the porphyrine, enzymatic HO-1 function can be activated (e.g. iron or cobalt atom) or, in the case of tin and zinc, inhibited $(19,20)$. Recently, we showed that in vivo administration of CoPP prior to conditioning resulted in prolonged survival and reduced aGvHD severity after experimental murine allogeneic BMT (21). As serum levels of interleukin 12 (IL-12) were markedly reduced in CoPP-treated animals, we hypothesized that CoPP treatment modulates APCs in this experimental setting. However, our results showed that CoPP treatment did not directly alter the stimulatory capacity of APCs and allospecific $\mathrm{T}$ cell stimulation, but rather mediated its protective effects by reducing the proinflammatory cytokine milieu after allogeneic BMT.

\section{Materials and methods}

Bone marrow transplantation (BMT). Female B6D2F1 (H-2 $\left.2^{\text {bxd }}\right)$, Balb/c (H-2 $\left.{ }^{\mathrm{d}}\right)$ and $\mathrm{C} 57 \mathrm{~B} 1 / 6\left(\mathrm{H}-2^{\mathrm{b}}\right)$ mice were purchased from Charles River Laboratories (Sulzbach, Germany) and were housed according to German animal protection laws and local institutional guidelines. Transplantation and all procedures were performed according to protocol approved by the local animal protection committee. Recipient animals received 12 or $14 \mathrm{~Gy}$ of total body irradiation split into two doses which were separated by a 4-h time interval to reduce gastrointestinal toxicity. Bone marrow was harvested from femurs and tibias of donor mice. Cell mixtures of erythrocyte-lysed $5 \times 10^{6}$ bone marrow cells and $5 \times 10^{6}$ splenic cells from syngeneic B6D2F1 or allogeneic $\mathrm{C} 57 \mathrm{Bl} / 6$ donors were re-suspended in Leibovitz L-15 medium (Gibco, Karlsruhe, Germany) and transplanted into B6D2F1 recipients via tail vein infusion $(0.25 \mathrm{ml}$ total volume). For HO-1 induction, recipient mice received $125 \mu \mathrm{g}$ CoPP intraperitoneally (i.p.) at day -2 and -1 before conditioning and transplantation. Control mice received phosphate-buffered saline (PBS) alone.

Assessment of GvHD. The severity of GvHD was assessed by a previously described clinical scoring system, which included 5 parameters: weight loss, posture, activity, fur texture and skin integrity (22). Mice were evaluated daily, and changes were graded from 0 to 2 for each criterion. A clinical index was subsequently generated by summation of the 5 criteria scores (maximum, 10). Animals showing a GvHD score $>6$ were sacrificed and counted as death due to aGHVD.

Induction of HO-1. For in vivo induction, CoPP (Frontier Scientific Products, Logan UT) was prepared under dim light because of the light sensitivity of the compound. CoPP was dissolved in $100 \mu \mathrm{l}$ sodium hydroxide $(150 \mathrm{mM})$, the $\mathrm{pH}$ was adjusted to 7.4 by adding hydrochloric acid and then a final concentration of $500 \mu \mathrm{g} / \mathrm{ml}$ was obtained by dilution with PBS. As previously described, otherwise untreated animals that received CoPP did not show any signs of toxicity such as hunching or reduction in mobility (21). For in vitro experiments the final concentration of CoPP was $20 \mu \mathrm{M}$.

\section{Cell preparation}

Peritoneal macrophages (MØ). Mice were sacrificed by $\mathrm{CO}_{2}$ asphyxiation and the peritoneal cavity was flushed subsequently with $5 \mathrm{ml}$ of ice-cold PBS. Cells were washed twice, counted and seeded in teflon bags.

Dendritic cells (DCs). Spleens were carefully dissolved between glass slides and filtered over a $70-\mu \mathrm{m}$ cell strainer (Falcon, Le Pont de Claix, France). Cells were incubated in PBS/0.5\% FCS and anti-CD11c microbeads and subsequently sorted according to the manufacturer's protocol (Miltenyi, Bergisch Gladbach, Germany). Purity was determined by flow cytometric analysis with directly labeled antibodies against CD11c and was generally $\sim 30-40 \%$.

T cells. For T cell isolation, splenic cells were incubated with anti-CD90 microbeads and isolated as described for DCs. Purity as determined by flow cytometry with directly labeled antibodies against CD4 and CD8 was generally $>85 \%$.

Antibodies and ELISA. Rabbit anti-mouse HO-1 (SPA-895) was purchased from StressGen (Victoria, Canada) and goat anti-rabbit IgG-FITC from Dianova (Hamburg, Germany). PE- or FITC-conjugated CD11c, CD80, CD86, H-2k, I-A, CD4 and CD8 antibodies were obtained from Becton Dickinson (Heidelberg, Germany). TNF $\alpha$ and IFN $\gamma$ ELISAs were obtained from Biosource (Nivelle, Belgium) and R\&D Systems (Wiesbaden, Germany), respectively. CD11c and CD90 microbeads were purchased from Miltenyi.

Flow cytometric analysis. Cells were washed in cold PBS/ $10 \%$ FCS and incubated on ice for 30 min with the $10 \%$ supernatant from clone $2.4 \mathrm{G} 2$ to block Fc $\gamma$ receptors. Then, FITC- or PE-conjugated antibodies (CD4, CD8, H-2k, I-A, CD80, CD86, CD11c) were added and incubated for $30 \mathrm{~min}$ on ice. Cells were washed twice with cold PBS/10\% FCS and analysed by FACSCalibur (Becton Dickinson).

For intracellular HO-1 staining, a protocol was used as described previously (23). Briefly, cells were fixed using $2 \%$ formaldehyde (Merck Eurolab, Darmstadt, Germany) for $10 \mathrm{~min}$ at $37^{\circ} \mathrm{C}$, next ice-cold methanol was added to a concentration of $90 \%$ and then the cells were incubated on ice for an additional $30 \mathrm{~min}$. After washing, cells were then labeled with rabbit anti-mouse HO-1 antibody and FITCconjugated goat anti-rabbit $\operatorname{IgG}$ secondary antibody for $15 \mathrm{~min}$ at room temperature. Additional surface staining was performed after intracellular staining.

Mixed lymphocyte reaction (MLR) and IFN $\gamma$ analysis. CD90+-sorted splenic T cells from C57Bl/6 mice $\left(\mathrm{H}-2 \mathrm{k}^{\mathrm{b}}\right)$ (200,000 per well) were plated in 96-well flat bottom plates. CD11 $\mathrm{c}^{+}$-sorted splenic dendritic cells from CoPP- or PBStreated allogeneic Balb/c mice $\left(\mathrm{H}-2 \mathrm{k}^{\mathrm{d}}\right)$ were irradiated (20 Gy) and then used as stimulators $(10,000$ per well) in the MLR. After $96 \mathrm{~h}$ of stimulation, ${ }^{3} \mathrm{H}$-thymidine was added to the culture, and after an additional $18 \mathrm{~h}$, proliferation activity 
A
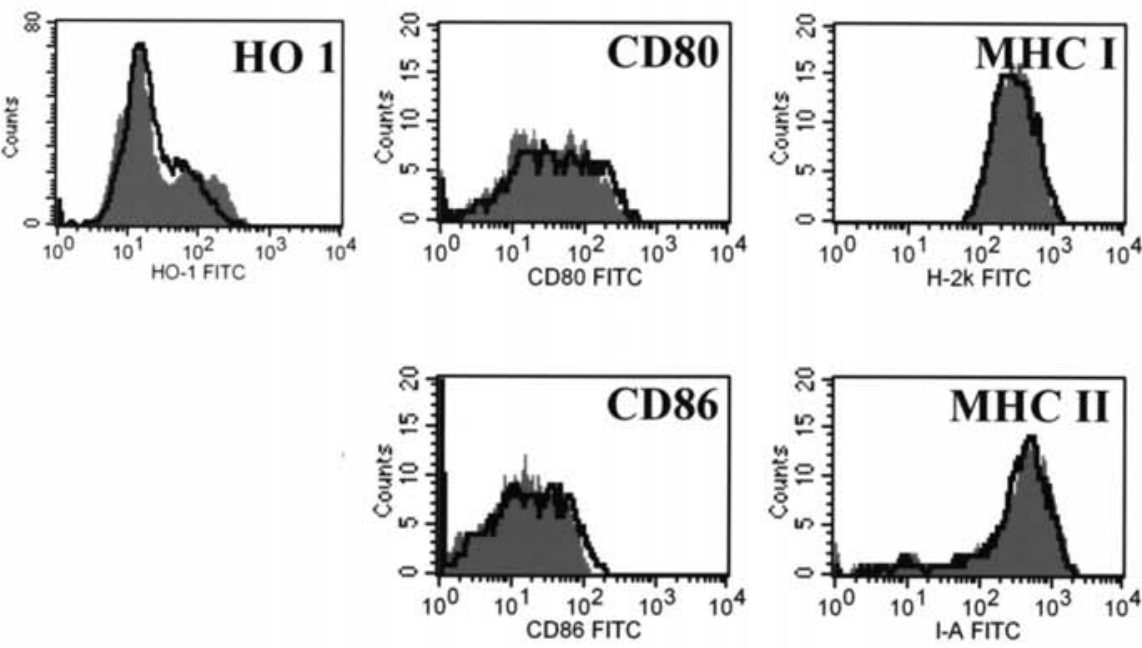

B in vitro proliferation

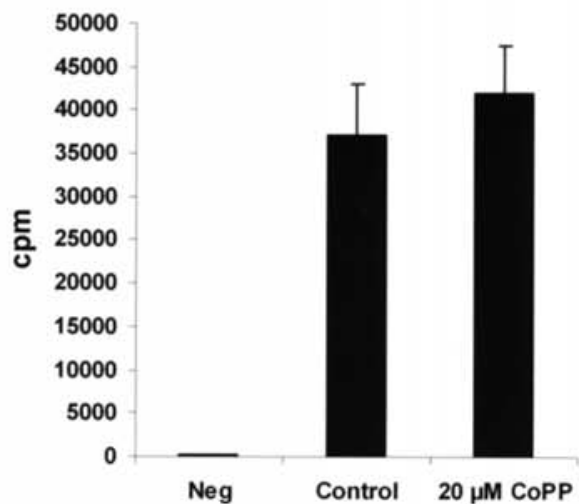

C

\section{IFN $\gamma$}

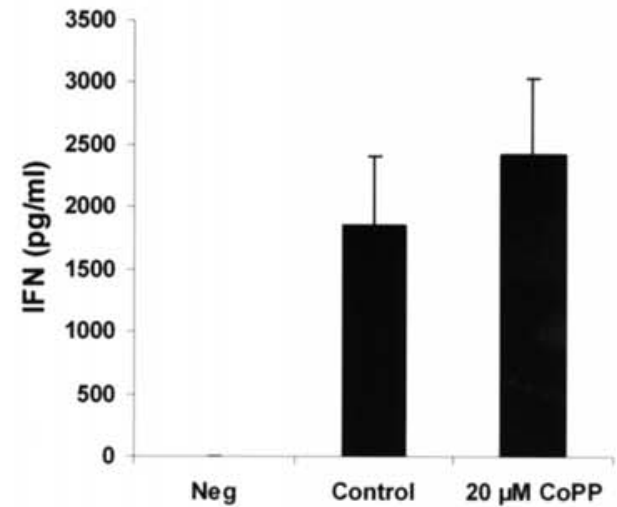

D
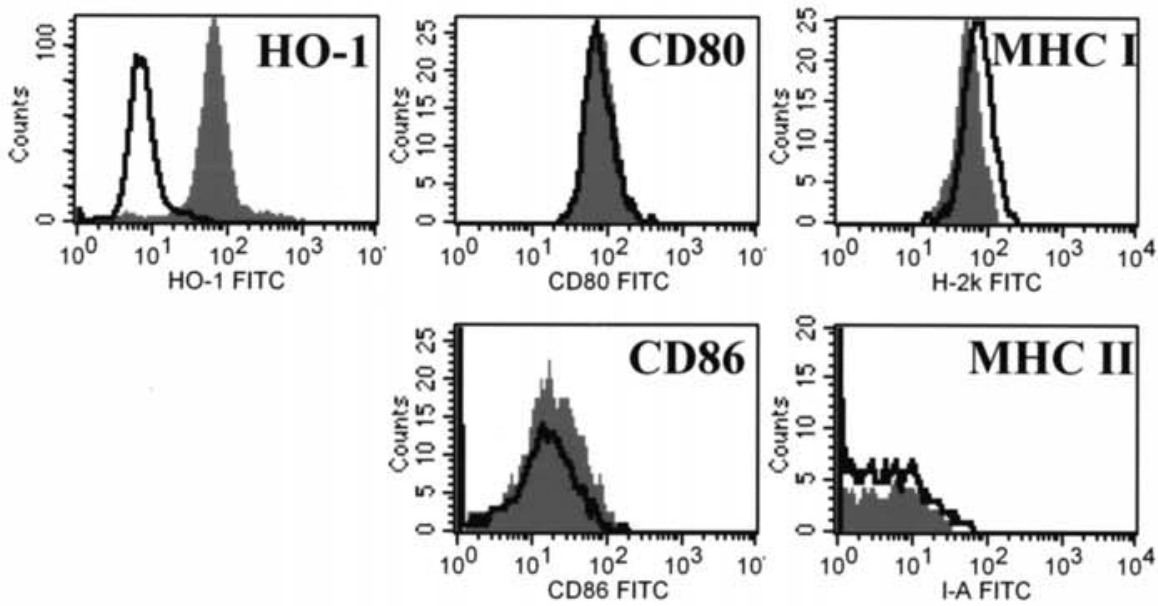

Figure 1. CoPP treatment did not enhance HO-1 expression in dendritic cells and did not alter the stimulatory capacity of DCs in mixed leukocyte reactions. Mice were treated with $125 \mu \mathrm{g}$ CoPP i.p. After $24 \mathrm{~h}$ DCs were isolated from the spleen as described in Materials and methods. (A) Cells were stained with unconjugated primary rabbit anti-mouse HO-1 antibody and secondary FITC-conjugated goat anti-rabbit IgG antibody, FITC-conjugated CD80, CD86, MHC class I or II antibody and PE-conjugated CD11c antibody and analysed by flow cytometry. The solid line represents control cells, the gray histogram indicates CoPP-treated cells. The data presented are from one of two comparable experiments. (B) CoPP-treated or untreated DCs were used as stimulators in a mixed lymphocyte reaction with CD90+ isolated, mismatched T cells. (C) Supernatants of the MLR were obtained prior to ${ }^{3} \mathrm{H}-\mathrm{Thymidine}$ labeling and used for IFN $\gamma$ levels. The data presented from (B) and (C) were run in a set of $15-18$ samples. (D) Isolated peritoneal macrophages were stimulated with or without $20 \mu \mathrm{M}$ CoPP in teflon bags for $24 \mathrm{~h}$ and were then analyzed by flow cytometry for expression of HO-1, CD80, CD86, MHC I and II. Solid lines represent control cells, and gray histograms indicate CoPP-treated cells. Data presented are from one of three comparable experiments. 
A

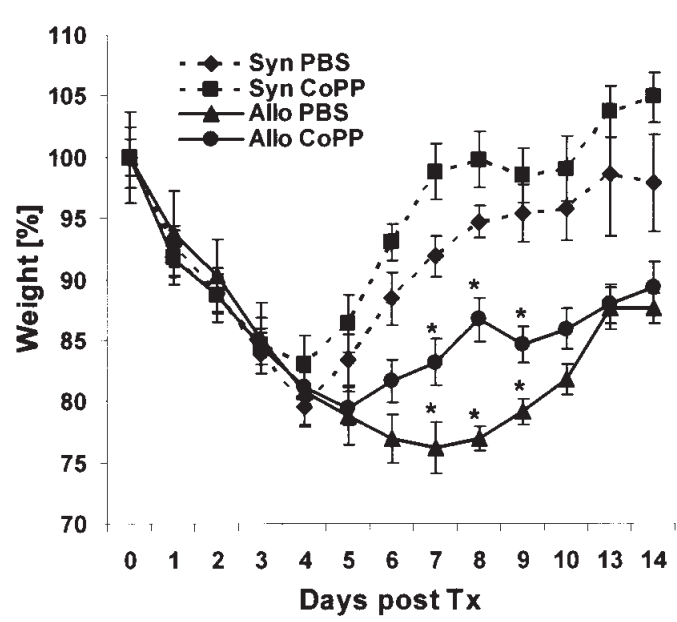

B

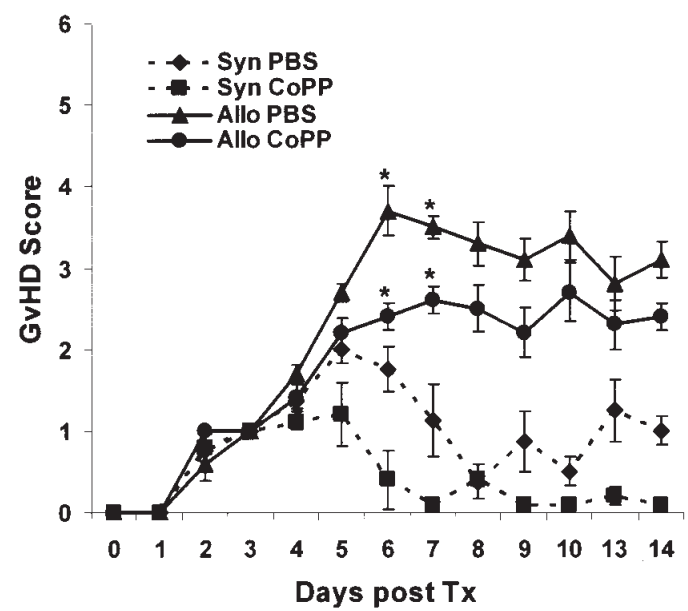

Figure 2. Reduction of acute GvHD after allogeneic BMT in CoPP-treated recipients. B6D2F1 mice were treated with CoPP or PBS on days -2 and -1 prior to 12 Gy irradiation, and BMT was performed with cells from either allogeneic (C57B1/6) or syngeneic (B6D2F1) donors on day 0. Mice were then monitored daily for weight (A) and GvHD score (B). (A) Treatment of B6D2F1 mice with CoPP (circle, solid line) prior to conditioning resulted in a faster weight recovery between day 6 and 10 compared to PBS-treated animals (triangle, solid line) after allogeneic BMT. Syngeneic groups (dotted lines) recovered faster than allogeneic groups. (B) CoPP treatment also resulted in a reduction of the clinical GvHD score between day 5 and 9 when compared with control animals after allogeneic BMT. The graphs show representative results from 1 of 2 independent experiments (5 animals per group). ${ }^{*}$ p<0.05 represents significant differences between PBS- and CoPP-treated animals.

was assessed by a microplate scintillation counter (Packard Canberra, Dreieich, Germany). Data were expressed as counts per min per well (mean \pm standard error). $\mathrm{T}$ cells stimulated with concanavalin A (Sigma) at a concentration of $4 \mu \mathrm{g} / \mathrm{ml}$ were used as positive control. Supernatants were obtained after $96 \mathrm{~h}$ and analysed for IFN $\gamma$ by ELISA according to the manufacturer's protocol.

$T$ cell expansion. Spleens were harvested 14 days after BMT. Cells were stained for CD4 and CD8 surface expression and analysed by flow cytometry. T cell expansion was calculated by the absolute number of splenic cells multiplied by the percentage of $\mathrm{CD}^{+}$or $\mathrm{CD}^{+}$positive cells in the cell suspension.

TNF $\alpha$ analysis. For determination of serum $\mathrm{TNF} \alpha$, blood samples were collected from animals 7-14 days after BMT. Serum supernatants were then harvested for analysis.

\section{Results}

In vivo administration of CoPP did not induce HO- 1 expression in splenic DCs and did not alter the alloantigenspecific $T$ cell response in mixed lymphocyte reactions after stimulation with CoPP-treated DCs. Dendritic cells are professional antigen-presenting cells that are believed to be responsible for initiating acute GvHD after allogeneic BMT $(1,24,25)$. As treatment of transplant recipients with CoPP prior to BMT reduced acute GvHD in a murine mouse model (21), we explored whether this effect was due to CoPP-induced changes in DC function and phenotype. Mice were treated with CoPP i.p. and DCs were isolated $24 \mathrm{~h}$ later from the spleen. HO-1 induction was not detected by flow cytometric analysis (Fig. 1A). In addition no significant difference in $\mathrm{T}$ cell proliferation was observed in the mixed lymphocyte reaction (MLR) when CoPP-treated and untreated splenic DCs were used as stimulators. Also, IFN $\gamma$ secretion did not differ in the supernatants from these MLR cultures (Fig. 1B and $\mathrm{C}$ ) indicating that the allostimulatory capacity of DCs was not changed by CoPP treatment.

CoPP treatment of macrophages in vitro resulted in an elevated HO-1 expression but did not affect the expression of costimulatory molecules such as CD80 and CD86. In addition, only a slight reduction of MHC class I and II expression was determined indicating that the activation status of the cells did not change significantly (Fig. 1D).

In vivo administration of CoPP reduced the severity of acute $G v H D$ after allogeneic BMT. To verify the protective effect of CoPP early after transplantation as seen in previous experiments from our group (21), B6D2F1 mice were treated with $125 \mu \mathrm{g}$ CoPP intraperitoneally for two days (day -2 and day -1 ) and then irradiated with 12 Gy (day 0 ) followed by the infusion of $5 \times 10^{6}$ bone marrow and $5 \times 10^{6}$ splenic cells of C57B1/6 mice. Syngeneic transplantation was performed with equal numbers of cells from B6D2F1 mice. Weight loss and clinical scores were evaluated daily for two weeks. As shown in Fig. 2A, both CoPP-treated and untreated syngeneic or allogeneic transplanted animals displayed weight losses of up to $20 \%$ on day four due to conditioning toxicity. On day five, syngeneic mice regained weight, while weight loss in allogeneic mice still increased. CoPP-treated allogeneic transplanted mice subsequently recovered earlier and faster than control mice (day 6-10) indicating that transplantationassociated toxicity and early acute GvHD were less severe in CoPP-treated animals compared to untreated animals after allogeneic BMT. This was confirmed by respective differences between groups using the clinical GvHD score, which incorporated changes in weight, fur, skin, mobility and 
A
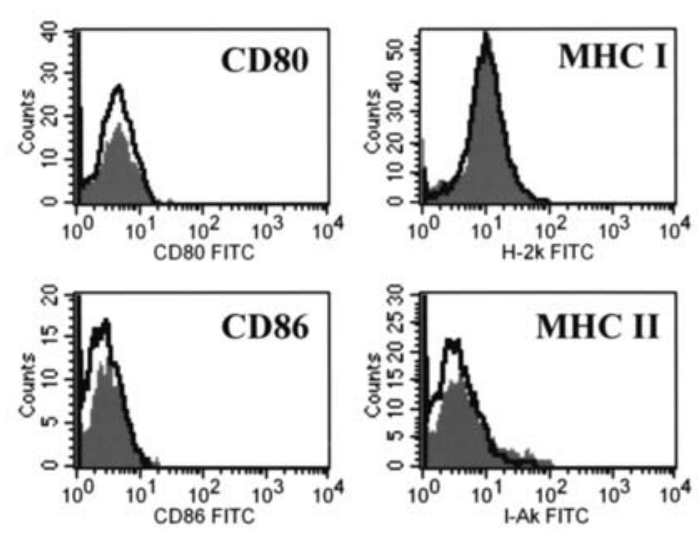

B

\section{CD4}

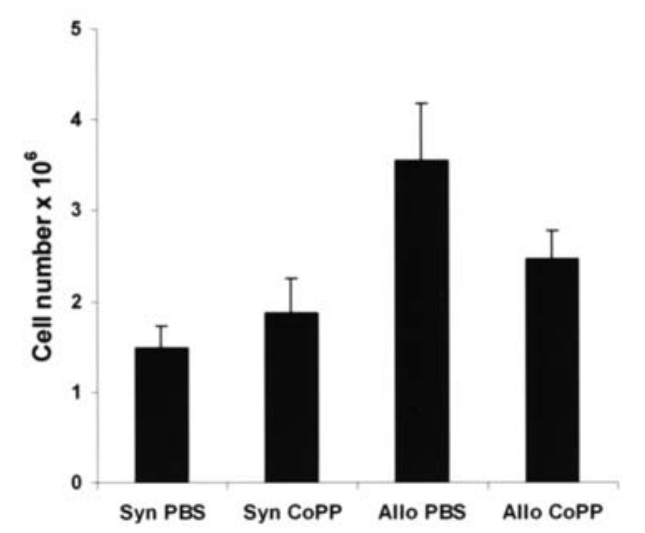

C

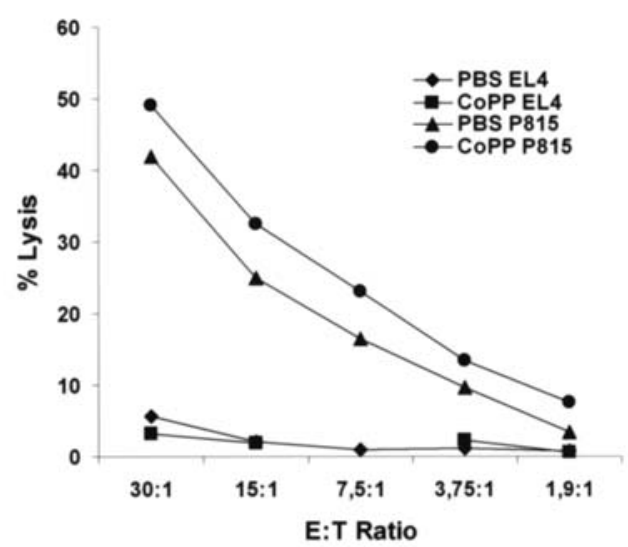

CD8

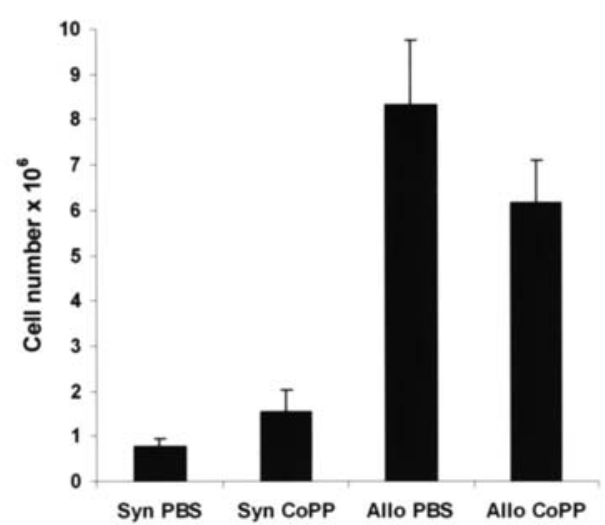

Figure 3. CoPP treatment did not alter the expression of costimulatory and MHC I and II molecules on dendritic cells or T cell expansion after allogeneic BMT. (A) B6D2F1 mice were treated with either CoPP or PBS and received BMT as described in Fig. 3. Between 1, 2 and 4 days after transplantation, DCs were isolated from the spleen and analyzed for CD80, CD86, MHC I and II by flow cytometry. Solid lines represent control cells, and gray histograms indicate CoPP-treated cells. The graphs show representative results for day 1 from 1 of 2 independent experiments. (B) Spleens were removed 14 days after allogeneic BMT and the numbers of $\mathrm{CD}^{+}$and $\mathrm{CD}^{+}$were analyzed by flow cytometry. Data are expressed as the means \pm SEM from 2 independent experiments. $n=8-12$ per group. (C) Spleens were removed 14 days after allogeneic BMT and isolated T cells were used in a cytotoxic T cell assay with EL4 as syngeneic and P815 as allogeneic targets. Shown is one of three comparable experiments.

posture (Fig. 2B). The total score of CoPP-treated allogeneic recipients rose to 2.5 while control animals reached a score of 4 on day six.

CoPP treatment of recipients neither altered in vivo dendritic cell activation nor in vivo $T$ cell expansion after allogeneic $B M T$. Having shown a lack of significant effects on costimulatory molecule expression and allostimulatory capacity of CoPP treatment on DCs in vitro, we next determined the effects of CoPP on the allostimulatory capacity of dendritic cells after allogeneic transplantation in vivo. Transplantation was performed as described above and splenic DCs were isolated on day 1,2 and 4 after transplantation. Flow cytometric analysis on day 1, 2 and 4 revealed that expression of CD80, CD86, MHC I and II was similar in cells from both the control and CoPP-treated allogeneic recipients (Fig. 3A). As activated APCs are responsible for $\mathrm{T}$ cell proliferation and activation after BMT, we determined the splenic $\mathrm{T}$ cell expansion on day 14 after transplantion. As shown in Fig. 3B, neither $\mathrm{CD}^{+}$nor $\mathrm{CD}^{+} \mathrm{T}$ cell expansion was significantly different after allogeneic BMT in the CoPP-treated and control groups indicating that CoPP does not affect the allospecific proliferative $\mathrm{T}$ cell response in vivo. In addition, no difference in the cytolytic capacity of $\mathrm{CD}^{+}$cells after BMT independent of CoPPpretreatment was observed (Fig. 3C).

CoPP treatment reduced TNF $\alpha$ serum levels after allogeneic $B M T$. As CoPP treatment did not affect the alloreactive T cell response both in vitro and in vivo, we extended our previous research (21) and tried to ascertain to what extent changes in weight loss may be due to differences in inflammatory cytokine production. We found that serum $\mathrm{TNF} \alpha$ was significantly reduced in CoPP-treated animals after allogeneic BMT (Fig. 4). These results reinforced our findings that the protective effects of CoPP-treatment in allogeneic BMT involve a reduction in the proinflammatory cytokine storm rather than alterations in allospecific $\mathrm{T}$ cell stimulation. 


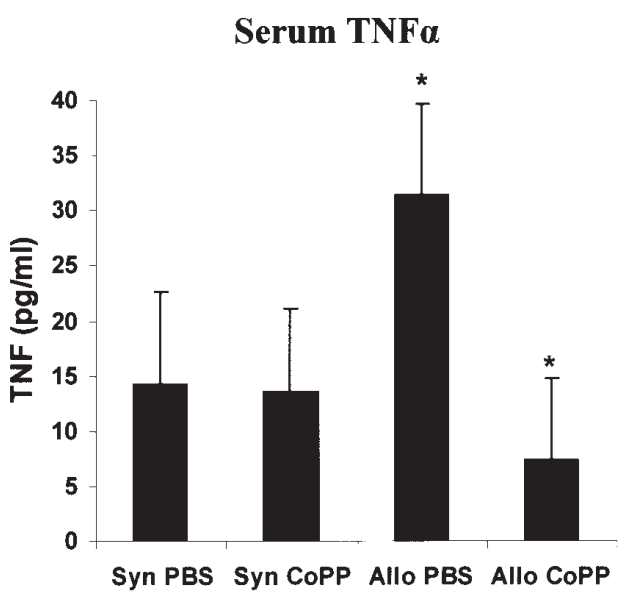

Figure 4. CoPP treatment prior to allogeneic BMT resulted in reduced serum $\mathrm{TNF} \alpha$. B6D2F1 mice were treated with either CoPP or PBS and received BMT as described in Fig. 3. Serum TNF $\alpha$ was analyzed on day 14 after transplantation by ELISA according to the manufacturer's protocol. Data are expressed as the means \pm SEM and are from two combined experiments $\left(\mathrm{n}=10\right.$ animals per group). ${ }^{*} \mathrm{p}=0.03$ represents significant differences between PBS- and CoPP-treated animals.

\section{Discussion}

The development of acute GvHD predominantly depends on the interactions of donor T cells with host APCs $(1,25,26)$ resulting in an alloantigen-specific $\mathrm{T}$ cell response against host tissues. T cell priming in this context is significantly influenced by the state of APC activation due to 'danger signals' during and after conditioning of the patient $(27,28)$. We have previously shown that in vivo administration of CoPP prior to allogeneic BMT leads to HO-1 induction, and to a reduction in clinical acute GvHD severity and to an overall improvement in survival (21). However, the exact mechanisms responsible for these observations have not been fully understood at this time.

In this study, we investigated the effects of CoPP treatment on alloantigen presentation in this context. We found that, rather than the modulation of $\mathrm{T}$ cell activation by APCs, a change in the early systemic proinflammatory cytokine milieu after allogeneic BMT seemed responsible for modulating GvHD severity and survival.

Consistent with in vitro data showing that $\mathrm{HO}-1$ expression is down-regulated in immature DCs by maturation stimuli such as lipopolysaccharide (LPS) and that mature DCs do not express HO-1 upon respective stimulation (29), administration of CoPP in vivo did not induce HO-1 expression in splenic DCs. Further, HO-1 induction in vivo did not alter the expression of MHC class I and II molecules and of costimulatory molecules such as CD80 and CD86 in these cells (Fig. 1A), consistent with results recently reported by Chauveau et al, who demonstrated no difference in CD80, CD86 and MHC I expression between untreated and CoPPtreated immature DCs as well as between CoPP-treated and LPS-matured human DCs in vitro (29). To exclude that this may be specific for dendritic cells, other professional APCs were also tested. MHC I, MHC II and costimulatory molecule expression were not altered in peritoneal macrophages despite CoPP-mediated HO-1 induction (Fig. 1D). It is well known that interactions of costimulatory molecules CD80/CD86 with T cell-expressed CD28 are necessary for T cell activation and expansion, and that inhibition of these interactions by using blocking antibodies against CD80 and CD86 results in reduced GvHD lethality (30). Along with no changes in costimulatory molecule expression, in vitro alloantigen-specific $\mathrm{T}$ cell stimulation by in vivo CoPPpretreated DCs was comparable to PBS-treated controls and resulted in comparable $\mathrm{T}$ cell proliferation and IFN $\gamma$ production both in our study (Fig. 1B and C) and as recently reported (29) for in vitro CoPP-pretreated DCs. Despite the indication that the in vitro allospecific $\mathrm{T}$ cell response against APCs, which were pretreated with $\mathrm{HO}$-1-inducing CoPP either in vitro or in vivo, is not altered significantly, in vivo $\mathrm{T}$ cell responses might differ slightly depending on the presence of other co-stimuli of APC activation such as LPS (28). This is supported by the fact that HO-1 induction prior to allogeneic BMT reduces irradiation-induced injury in radiation-sensitive organs such as the small bowel, which is associated with significant reduction in serum LPS levels (21). LPS has been shown to activate APCs and to enhance alloreactive $\mathrm{T}$ cell responses (31-33). This has been recently confirmed by Chauveau et al (29). The authors were able to demonstrate an increased allogeneic stimulatory capacity for LPS-treated DCs, which was significantly reduced after a previous co-culture of DCs with LPS and CoPP, therefore suggesting an inhibitory effect of HO-1-induction in APCs on $\mathrm{T}$ cell activation in the presence of APC-activating costimuli. LPS further plays an essential role in the pathophysiology of graft-versus-host disease (34) by mediation of the inflammatory cytokine production early after transplant and subsequent regulation of the cellular effector phase later during disease development.

Concordant with the in vitro experiments, our in vivo results showed that neither $\mathrm{CD}^{+}{ }^{+}$nor $\mathrm{CD}^{+}{ }^{+} \mathrm{T}$ cell expansion was significantly different between the CoPP-treated and control groups 14 days after allogeneic BMT. However, T cells from CoPP-treated animals after allogeneic BMT seemed to have a tendency towards less expansion in secondary lymphoid tissues (Fig. 3B), not ruling out that a reduced expression of other co-stimuli might have contributed to this effect.

It has been shown in various experimental studies, that HO-1 expression can directly suppress $\mathrm{T}$ cell proliferation $(35,36)$. However, in these models $\mathrm{T}$ cells overexpressed HO-1 themselves or encountered CoPP directly with or without the presence of APCs. In our experimental setting, though, $\mathrm{HO}-1$ expression was not primarily induced in $\mathrm{T}$ cells through CoPP treatment. Instead, T cells were inoculated into host mice, which were treated with CoPP two days prior to T cell administration. Therefore it seems most likely that, rather than reflecting CoPP-mediated effects directly on donor $\mathrm{T}$ cells, the observed slight suppression of $\mathrm{T}$ cell proliferation was primarily due to effects of CoPP-pretreatment on APCs, which became evident in the context of a pro-inflammatory microenvironment after lethal irradiation conditioning. The possibility that residual CoPP traces had a direct effect on the administered $\mathrm{T}$ cells and their proliferative potential, however, can not be totally excluded.

The pathophysiology of GvHD can be divided into different phases including an early inflammatory cytokine 
response and a later occurring cytotoxic cellular effector phase. While the former significantly contributes to early mortality after transplant (37), the latter predominantly involves target tissue injury by infiltrating alloreactive $\mathrm{T}$ cells (38). As neither donor T cell proliferation nor cytotoxic $\mathrm{T}$ cell function were significantly altered in allogeneic recipients pretreated with CoPP (Fig. 3C), other factors seem responsible for the previously reported (21) decreased GvHD and improved survival after allogeneic BMT.

One of the major cytokines during the early inflammatory phase after allogeneic BMT is $\mathrm{TNF} \alpha$. TNF $\alpha$ levels and interactions between TNF $\alpha$ and TNF receptor type I (TNFR I) are directly associated with aGvHD severity and early mortality after allogeneic BMT both in clinical and experimental studies $(4,34,39)$. We previously demonstrated significantly reduced systemic TNF $\alpha$ levels 7 days after allogeneic BMT in CoPPtreated animals (21). To extend these results, we tested whether the reduction in serum $\mathrm{TNF} \alpha$ was persistent over a prolonged time period after BMT. TNF $\alpha$ levels were still decreased even 14 days after transplant (Fig. 4) and, as expected, reduced TNF $\alpha$ levels led to less aGvHD (Fig. 2). As we previously published the survival benefit in response to CoPP pretreatment of allogeneic recipients in this model (21), in this study survival experiments were not conducted. The scientific contribution to this manuscript of another set of survival experiments using living animals would have been only marginal and was not justified by German animal protection law.

In summary, CoPP-mediated HO-1 induction in allogeneic recipients results in improved survival and decreased clinical GvHD severity. As an extension of previous research from our group (21), this study demonstrates that, rather than alterations in the alloantigen-specific $\mathrm{T}$ cell response, a downmodulation of the early inflammatory cytokine production by host macrophages, specifically of $\mathrm{TNF} \alpha$ as one of the key player cytokines in GvHD pathophysiology, seems responsible. Further studies are necessary to elucidate the further role of HO-1 induction in altering the crosslinking between the adaptive and innate immune response after allogeneic BMT.

\section{Acknowledgements}

This study was supported by the Wilhelm Sander Foundation grant no. 2002.120.1 and in part by the Rudolf Bartling Foundation.

\section{References}

1. Shlomchik WD, Couzens MS, Tang CB, et al: Prevention of graft versus host disease by inactivation of host antigenpresenting cells. Science 285: 412-415, 1999.

2. Antin JH and Ferrara JL: Cytokine dysregulation and acute graft-versus-host disease. Blood 80: 2964-2968, 1992.

3. Ferrara JL: Cytokine dysregulation as a mechanism of graft versus host disease. Curr Opin Immunol 5: 794-799, 1993.

4. Holler E, Kolb HJ, Mittermuller J, et al: Modulation of acute graft-versus-host-disease after allogeneic bone marrow transplantation by tumor necrosis factor alpha (TNF alpha) release in the course of pretransplant conditioning: role of conditioning regimens and prophylactic application of a monoclonal antibody neutralizing human TNF alpha (MAK 195F). Blood 86: 890-899, 1995.

5. Holler E, Kolb HJ, Eissner G and Wilmanns W: Cytokines in GvH and GvL. Bone Marrow Transplant 22 (suppl 4): 3-6, 1998.
6. Cooke KR, Olkiewicz K, Erickson N and Ferrara JL: The role of endotoxin and the innate immune response in the pathophysiology of acute graft versus host disease. J Endotoxin Res 8: 441-448, 2002.

7. Wagener FA, Eggert A, Boerman OC, et al: Heme is a potent inducer of inflammation in mice and is counteracted by heme oxygenase. Blood 98: 1802-1811, 2001.

8. Bouton C and Demple B: Nitric oxide-inducible expression of heme oxygenase-1 in human cells. Translation-independent stabilization of the mRNA and evidence for direct action of nitric oxide. J Biol Chem 275: 32688-32693, 2000.

9. Sugishima M, Omata Y, Kakuta Y, Sakamoto H, Noguchi M and Fukuyama K: Crystal structure of rat heme oxygenase- 1 in complex with heme. FEBS Lett 471: 61-66, 2000.

10. McCoubrey WK Jr, Huang TJ and Maines MD: Isolation and characterization of a cDNA from the rat brain that encodes hemoprotein heme oxygenase-3. Eur J Biochem 247: 725-732, 1997.

11. Erario MA, Gonzales S, Noriega GO and Tomaro ML: Bilirubin and ferritin as protectors against hemin-induced oxidative stress in rat liver. Cell Mol Biol (Noisy-le-grand) 48: 877-884, 2002.

12. Ferris CD, Jaffrey SR, Sawa A, et al: Haem oxygenase-1 prevents cell death by regulating cellular iron. Nat Cell Biol 1: 152-157, 1999.

13. Zhang X, Shan P, Otterbein LE, et al: Carbon monoxide inhibition of apoptosis during ischemia-reperfusion lung injury is dependent on the p38 mitogen-activated protein kinase pathway and involves caspase 3. J Biol Chem 278: 1248-1258, 2003.

14. Brouard S, Otterbein LE, Anrather J, Tobiasch E, Bach FH, Choi AM and Soares MP: Carbon monoxide generated by heme oxygenase 1 suppresses endothelial cell apoptosis. J Exp Med 192: 1015-1026, 2000.

15. Lee TS and Chau LY: Heme oxygenase- 1 mediates the antiinflammatory effect of interleukin-10 in mice. Nat Med 8: 240-246, 2002.

16. Soares MP, Lin Y, Anrather J, et al: Expression of heme oxygenase- 1 can determine cardiac xenograft survival. Nat Med 4: 1073-1077, 1998

17. Petrache I, Otterbein LE, Alam J, Wiegand GW and Choi AM: Heme oxygenase- 1 inhibits TNF-alpha-induced apoptosis in cultured fibroblasts. Am J Physiol Lung Cell Mol Physiol 278: L312-L319, 2000.

18. Reeve VE and Tyrrell RM: Heme oxygenase induction mediates the photoimmunoprotective activity of UVA radiation in the mouse. Proc Natl Acad Sci USA 96: 9317-9321, 1999.

19. Katori M, Buelow R, Ke B, et al: Heme oxygenase-1 overexpression protects rat hearts from cold ischemia/reperfusion injury via an antiapoptotic pathway. Transplantation 73: 287-292, 2002 .

20. Sass G, Soares MC, Yamashita K, et al: Heme oxygenase-1 and its reaction product, carbon monoxide, prevent inflammationrelated apoptotic liver damage in mice. Hepatology 38: 909-918, 2003.

21. Gerbitz A, Ewing P, Wilke A, et al: Induction of heme oxygenase-1 before conditioning results in improved survival and reduced graft-versus-host disease after experimental allogeneic bone marrow transplantation. Biol Blood Marrow Transplant 10: 461-472, 2004.

22. Cooke KR, Kobzik L, Martin TR, Brewer J, Delmonte J Jr, Crawford JM and Ferrara JL: An experimental model of idiopathic pneumonia syndrome after bone marrow transplantation: I. The roles of minor $\mathrm{H}$ antigens and endotoxin. Blood 88: 3230-3239, 1996.

23. Chow S, Patel H and Hedley DW: Measurement of MAP kinase activation by flow cytometry using phospho-specific antibodies to MEK and ERK: potential for pharmacodynamic monitoring of signal transduction inhibitors. Cytometry 46: 72-78, 2001.

24. Duffner UA, Maeda Y, Cooke KR, et al: Host dendritic cells alone are sufficient to initiate acute graft-versus-host disease. J Immunol 172: 7393-7398, 2004.

25. Teshima T, Ordemann R, Reddy P, Gagin S, Liu C, Cooke KR and Ferrara JL: Acute graft-versus-host disease does not require alloantigen expression on host epithelium. Nat Med 8: 575-581, 2002.

26. Li JM and Waller EK: Donor antigen-presenting cells regulate T-cell expansion and antitumor activity after allogeneic bone marrow transplantation. Biol Blood Marrow Transplant 10: 540-551, 2004. 
27. Matzinger P: The danger model: a renewed sense of self. Science 296: 301-305, 2002.

28. Reddy P: Pathophysiology of acute graft-versus-host disease. Hematol Oncol 21: 149-161, 2003.

29. Chauveau C, Remy S, Royer PJ, et al: Heme oxygenase-1 expression inhibits dendritic cell maturation and proinflammatory function but conserves IL-10 expression. Blood 106: 1694-1702, 2005.

30. Blazar BR, Sharpe AH, Taylor PA, Panoskaltsis-Mortari A, Gray GS, Korngold R and Vallera DA: Infusion of anti-B7.1 (CD80) and anti-B7.2 (CD86) monoclonal antibodies inhibits murine graft-versus-host disease lethality in part via direct effects on CD4+ and CD8+ T cells. J Immunol 157: 3250-3259, 1996.

31. Tough DF, Sun S and Sprent J: T cell stimulation in vivo by lipopolysaccharide (LPS). J Exp Med 185: 2089-2094, 1997.

32. Banchereau J and Steinman RM: Dendritic cells and the control of immunity. Nature 392: 245-252, 1998.

33. Sallusto F and Lanzavecchia A: The instructive role of dendritic cells on T-cell responses. Arthritis Res 4 (suppl 3): 127-132, 2002.
34. Cooke KR, Hill GR, Crawford JM, Bungard D, Brinson YS, Delmonte J Jr and Ferrara JL: Tumor necrosis factor-alpha production to lipopolysaccharide stimulation by donor cells predicts the severity of experimental acute graft-versus-host disease. J Clin Invest 102: 1882-1891, 1998.

35. Braudeau C, Bouchet D, Tesson L, et al: Induction of long-term cardiac allograft survival by heme oxygenase- 1 gene transfer. Gene Ther 11: 701-710, 2004

36. Pae HO, Oh GS, Choi BM, Chae SC, Kim YM, Chung KR and Chung HT: Carbon monoxide produced by heme oxygenase-1 suppresses T cell proliferation via inhibition of IL-2 production. J Immunol 172: 4744-4751, 2004.

37. Speiser DE, Bachmann MF, Frick TW, et al: TNF receptor p55 controls early acute graft-versus-host disease. J Immunol 158: 5185-5190, 1997.

38. Reddy P and Ferrara JL: Immunobiology of acute graft-versushost disease. Blood Rev 17: 187-194, 2003.

39. Hill GR, Crawford JM, Cooke KR, Brinson YS, Pan L and Ferrara JL: Total body irradiation and acute graft-versus-host disease: the role of gastrointestinal damage and inflammatory cytokines. Blood 90: 3204-3213, 1997. 